\title{
Structures of the Oligosaccharides from the Enzymic Hydrolyzate of Rice-straw Arabinoxylan by a Xylanase of Aspergillus niger ${ }^{\dagger}$
}

\author{
Shigeyuki TAKenisHI and Yoshio TsuJISAKa \\ Osaka Municipal Technical Research Institute, \\ Kitaogimachi, Kita-ku, Osaka, Japan \\ Received November 10, 1972
}

\begin{abstract}
A trisaccharide consisting of two D-xylose units and one L-arabinose unit, and a tetrasaccharide consisting of three D-xylose units and one L-arabinose unit were isolated from the hydrolyzate of rice-straw arabinoxylan by the xylanase I produced by Asp. niger.

The structures of the trisaccharide and the tetrasaccharide were determined to be $3^{1}-\alpha$ L-arabinofuranosylxylobiose $\left([a]_{D^{-}} 80^{\circ}\right)$ and $3^{1}-a$-L-arabinofuranosylxylotriose $\left([a]_{D^{-}} 84^{\circ}\right)$, respectively, by chemical and enzymic methods.

According to the structures of two arabinose-xylose mixed oligosaccharides, it was shown that the rice-straw arabinoxylan is composed of chain of 1,4-linked $\beta$-D-xylopyranose residues and some of xylose residues have side-chain of 1,3-linked $a$-L-arabinofuranose.
\end{abstract}

As previously reported, ${ }^{1 \sim 2)}$ it was found that a strain of $A s p$. niger produces three xylanases (named I, II and III) on the wheat bran koji culture. These enzymes were highly purified and some properties and specificities on substrates were investigated.

From the investgation, it appeared that these xylanases are different from each other in the degree of hydrolysis on various substrates and hydrolytic products from arabinoxylan.

It was previously found that the xylanase I produces the xylobiose and a series of oligosaccharides consisting of both arabinose and xylose units in fairly large quantities. Of these oligosaccharides, one was a trisaccharide consisting of one arabinose unit and two xylose units, and the other was a tetrasaccharide also consisting of one arabinose unit and three xylose units.

Several reports have been published on the determination of the structure of oligosaccharides in which arabinose and xylose units were combined.

C. T. Bishop ${ }^{3)}$ isolated a trisaccharide con-

$\uparrow$ Studies on Hemicellulases. Part V. See reference $^{1,2,6)}$ and $Y$. Tsujisaka, K. Hiyama, S. Takenishi and J. Fukumoto, Nippon Nôgeikagaku Kaishi, 46, 155 (1972). sisting of one arabinose unit and two xylose units from wheat-straw xylan by hydrolysis with a purified cellulolytic enzyme preparation from Myrothecium verrucaria, and determined its structure to be $3^{2}$ - $(\alpha$ or $\beta$ )-L-arabinofuranosylxylobiose $\left([a]_{\mathrm{D}}-15^{\circ}\right)$ by the chemical method.

G. O. Aspinall et al. ${ }^{4)}$ also isolated the same trisaccharide from the enzymic digests of rye flour and cooksfoot grass arabinoxylan.

H. R. Goldshmid and A. S. Perlin ${ }^{5 \text { ? }}$ isolated a tetrasaccharide consisting of both arabinose and xylose units from the enzymic digests of the soluble arabinoxylan of wheat by an enzyme preparation obtained from Streptmyces QMB 814 , and determined the structure to be $3^{2}-a$ L-arabinofuranosylxylotriose $\left([a]_{D}-75^{\circ}\right)$.

However, the results of the specific rotatory power and mobility on paper chromatography of the oligosaccharides produced by the xylanase I did not agree with the results obtained by the other authors.

The present paper deals with the investigations for clarifing the structures of these two oligosaccharides by the chemical method of periodate oxidation and the enzymic method using the specificities of the $\beta$-xylosidase and the xylanase II obtained from Asp. niger. 


\section{MATERIALS AND METHODS}

Enzyme preparations. The xylanase I, II and the $\beta$-xylosidase were prepared from the extract of koji culture of Asp. niger by the procedures described previously.1,6) The preparations of the xylanase I and the xylanase II were homogeneouse on ultracentrifugal and electrophoretic analyses. The preparation of $\beta$-xylosidase was purified in a state free from xylanase and arabinofuranosidase.

Materials. Rice-straw arabinoxylan was prepared by the procedures described previously. " Phenyl $a$ L-arabinofuranoside was kindly supplied by Prof. A. Kaji, Kagawa University. Phenyl $\beta$-L-arabinofuranoside was purchased from Nakarai Chemicals Ltd.

Enzymic degradation of rice-straw arabinoxylan. Reaction mixture contained $5 \mathrm{~g}$ of rice-straw arabinoxylan and $10 \mathrm{mg}$ of the xylanase $I$ in $500 \mathrm{ml}$ of $0.02 \mathrm{~m}$ acetate buffer, $\mathrm{pH} 4.5$, and was incubated at $40^{\circ} \mathrm{C}$ for $48 \mathrm{hr}$. By this treatment, the reducing power corresponding to $53 \%$ convertion into xylose was obtained. After the insoluble matters were centrifuged off, the supernatant was concentrated to a volume of $30 \mathrm{ml}$ under reduced pressure at $40 \sim 45^{\circ} \mathrm{C}$.

Isolation of oligosaccharides. Oligosaccharides were isolated from the hydrolyzate of arabinoxylan by the following procedures.

Thirty milliliter of the concentrate mentioned above was chromatographed through a column of Sephadex G-15 $(4 \times 120 \mathrm{~cm})$ washed throughly with deionized water. The elution was carried out with deionized water at a flow rate of $25 \mathrm{ml}$ per hr.

By this chromatography, the hydrolyzate was divided into four fractions such as monosaccharide, disaccharide, trisaccharide and highly polymerized sugars. Each fraction was further purified by preparative chromatography on Whatman $3 \mathrm{MM}$ paper ${ }^{7}$ and lyophilized.

Determination and identification of component sugars of oligosaccharides. About $2 \mathrm{mg}$ of oligosaccharide was hydrolyzed with $0.5 \mathrm{ml}$ of $1 \mathrm{~N}$ sulfuric acid in a small sealed tube $(0.8 \times 6 \mathrm{~cm})$ at $100 \mathrm{C}$ for $2 \mathrm{hr}$. The hydrolyzate was neutralized with sodium hydroxide and deionized with a column of mixed ion-exchange resins (Diaion $\mathrm{SK} 1 \mathrm{~B} \mathrm{H}^{+}$and $\mathrm{SA} 10 \mathrm{~A} \mathrm{OH}^{-}$). Deionized solution was concentrated to a volume of $0.1 \mathrm{ml}$ under reduced pressure at $40 \sim 45^{\circ} \mathrm{C}$. Adequate amount of the concentrate was spotted on Whatman No. 1 filter paper and chromatographed by the method described later. The hydrolysis products were identified by comparing their $R f$ values with those of the authentic sugars, and the color density was measured at $460 \mathrm{~m} \mu$ in a densitometer (Joyce-Loeble, Chromos- can). The amount of sugars were calculated by the calibration graph which had been prepared with given quantity of arabinose and xylose.

Estimation of degree of polymerization of oligosaccharide. Degree of polymerization of oligosaccharide was estimated by the method of Timell as follows. ${ }^{8}$

The oligosaccharide $(0.5 \sim 1.0 \mathrm{mg})$ was dissolved in $10 \mathrm{ml}$ of deionized water. One milliliter aliquots were added to six test tubes $(18 \times 180 \mathrm{~mm})$ provided with ground-grass stoppers. Water $(1 \mathrm{ml})$ was added to one similar tube to serve as blank. Active sodium borohydride solution $(0.5 \mathrm{ml}, 1 \%$ in deionized water) was added to each of the first three sugar solutions and reduction at reducining ends was proceeded at $15^{\circ} \mathrm{C}$. After $24 \mathrm{hr}, 0.5 \mathrm{ml}$ of inactive borohydride solution ( $1 \%$ in $1 \mathrm{~N}$ sulfuric acid) was added to the remaining four test tubes (three sugar solutions and one blank) and amount of sugar in each test tube was measured by phenol-sulfuric acid method. ${ }^{91}$

If $A$ is the amount of total sugar of the parent oligosaccharide (before reduction) and $B$ is that of after the reduction, degree of polymerization of oligosaccharide (D.P.) can be calculated as follows:

$$
\text { D.P. }=\mathrm{A} / \mathrm{A}-\mathrm{B}
$$

Estimation of specific rotatory power of oligosaccharides. Specific rotatory power of oligosaccharide was measured in the aqueous solution $(1 \%$ or $2 \%)$ at $20^{\circ} \mathrm{C}$ with an automatic polarimeter (Japan Spectroscopic Co. Ltd., Model DIP-SL).

Paper chromatography. Paper chromatography was carried out on Whatman No. 1 filter paper for qualitative and quantitative chromatography or Whatman $3 \mathrm{MM}$ filter paper for preparative chromatography using the solvent system of $n$-butanol: pyridine: water $(6: 4: 2.5 \mathrm{v} / \mathrm{v})$ by the multiple developments of the ascending method.

Sugars were detected on the paper by silver nitrate. ${ }^{101}$ Aniline hydrogen phthalate was also used for a detection of reducing sugars. ${ }^{11}$

Reduction of oligosaccharides by sodium borohydride. Each oligosaccharide was dissolved in deionized water up to the concentration of $0.01 \mathrm{M}$. One milliliter of $0.5 \mathrm{M}$ sodium borohydride was added to $10 \mathrm{ml}$ of the oligosaccharide solution and kept at $15^{\circ} \mathrm{C}$ for $24 \mathrm{hr}$. After the excess amount of sodium borohydride was destroyed by adding the suitable quantity of glacial acetic acid, the solution was loaded on a column of mixed ion-exchange resins (Diaion $\mathrm{SK} 1 \mathrm{~B} \mathrm{H}^{+}$and SA $\left.10 \mathrm{~A} \mathrm{OH}^{-}\right)$and effluent was concentrated to a volume of $2 \mathrm{ml}$ under reduced pressure at $40 \sim 45^{\circ} \mathrm{C}$ and lyophilized.

Oxidation of oligosaccharides by sodium metaperiodate. Reaction mixture containing $10 \mathrm{ml}$ of $0.01 \mathrm{M}$ 
of oligosaccharide and $10 \mathrm{ml}$ of $0.1 \mathrm{M}$ of sodium metaperiodate was kept at $7^{\circ} \mathrm{C}$ in the dark room. Aliquots of the mixture were periodically withdrawn and the amounts of periodate consumed and formic acid produced were measured by the method of FleuryLange $^{12)}$ and by the means of titration with sodium hydroxide using methyl red as indicator, respectively.

Enzymic degradation of oligosaccharides by the xylanase II or the B-xylosidase of Asp. niger. Reaction mixture containing $1 \mathrm{mg}$ of oligosaccharide which was dissolved in $0.1 \mathrm{ml}$ of deionized water and $0.01 \mathrm{ml}$ of the enzyme solution containing $0.1 \mathrm{mg}$ of the $\beta$ xylosidase or the xylanase II was incubated at $40^{\circ} \mathrm{C}$ for $24 \mathrm{hr}$.

\section{RESULTS}

Oligosaccharides produced from rice-straw arabinoxylan by the xylanase I

Isolation of oligosaccharides produced from rice-straw arabinoxylan by hydrolyses with three xylanases of $A s p$. niger were described in the previous paper. ${ }^{2}$

In this paper, the results of analyses of the oligosaccharides produced from rice-straw arabinoxylan by the xylanase I were shown in Fig. 1 and Table $I$. Each oligosaccharide was named as shown in Fig. 1.

As indicated in the previous paper, ${ }^{2}$ it was found that out of four oligosaccharides, oligosaccharide I-2 was xylobiose ( $O$ - $\beta$-Dxylopyranosyl-( $1 \rightarrow 4)$-D-xylopyranose).

Reduction of oligosaccharide $I-1$ and oligosaccharide I-3 by sodium borohydride and hydrolysis of reduced oligosaccharides

Oligosaccharide I-1 and I-3 were reduced by the method described in MATERIALS AND METHODS.

On acid hydrolysis, the reduced oligosaccharide I-1 gave arabinose and xylose and it was confirmed by densitometric method described in MATERIALS AND METHODS that they were in the ratio $1: 1$. The reduced oligosaccharide I-3 gave arabinose and xylose in the proportion of $1: 2$ on acid hydrolysis (Fig. 2). Namely, both oligosaccharides lost one xylose residue on the reduction by sodium borohydride.
Table I. Results of Analyses of IsOLATEd OLIGOSACCHARIDES

A series of oligosaccharides were isolated from products of the xylanase I by chromatographic fractionations on a column of Sephadex $G-15$ and preparative paper chromatography. See the text for experimental detail.

\begin{tabular}{ccccc}
\hline $\begin{array}{c}\text { Oligosac- } \\
\text { charide }\end{array}$ & \multicolumn{2}{c}{ Component sugars } & $\begin{array}{c}\text { Degree } \\
\text { of } \\
\text { polyme- } \\
\text { rization }\end{array}$ & $\begin{array}{c}\text { Specific } \\
\text { rotatory } \\
\text { value } \\
{[\alpha]_{\mathrm{D}}^{20}}\end{array}$ \\
\hline I-1 & 2 & 1 & 3 & $-80^{\circ}(c=1)$ \\
I-2 & + & - & 2 & $-27^{\circ}(c=2)$ \\
I-3 & 3 & 1 & 4 & $-84^{\circ}(c=1)$ \\
I-4 & 4 & 1 & 5 & - \\
\hline
\end{tabular}

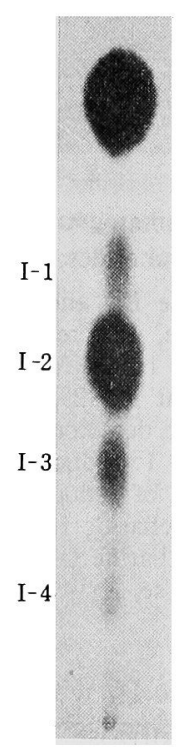

FIG. 1. Paper Chromatogram of Products of the Xylanase I from Rice-straw Arabinoxylan.

Each product was named as shown in the figure. The experimental details are described in the text. Paper chromatography was carried out by the method described in the text and alkaline silver nitrate was used as a detector.

On enzymic hydrolysis by the $\beta$-xylosidase of Asp. niger, the reduced oligosaccharide I-1 and I-3 gave only xylose as reducing sugars (Fig. 3).

Enzymic degradation of oligosaccharide $I-1$ and oligosaccharide I-3 by the xylanase II of Asp. niger

As shown in Fig. 4, arabinose and xylobiose were found in the digest of oligosacchride 


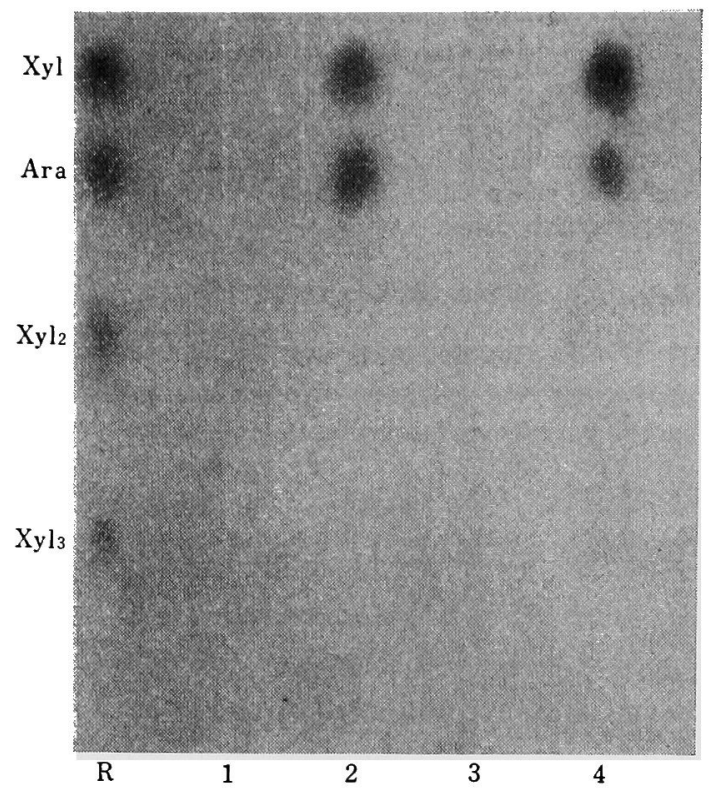

FIG. 2. Paper Chromatogram of Acid Hydrolyzates of Reduced Oligosaccharides.

After oligosaccharide I-1 and I-3 were reduced by sodium borohydride, the reduced oligosaccharides were hydrolyzed by $1 \mathrm{~N}$ sulfuric acid. See the text for the experimental details. Aniline hydrogenphtharate was used as a detector.

$\mathrm{R}$ : reference sugars, 1 : reduced oligosaccharide $\mathrm{I}-1$, 2: acid hydrolyzate of reduced oligosaccharide I-1, 3: reduced oligosaccharide I-3, 4: acid hydrolyzate of reduced oligosaccharide I-3, Ara: arabinose, Xyl: xylose, $\mathrm{Xyl}_{2}$ : xylobiose, $\mathrm{Xyl}_{3}$ : xylotriose.

I-1 by the xylanase II, while arabinose, xylose, xylobiose and xylotriose were detected in the digest of oligosaccharide I-3.

\section{Oxidation of oligosaccharide $I-1$ and oligosac- charide $I-3$ by sodium meatperiodate}

The oligosaccharide I-1 was oxidized by sodium metaperiodate by the method described in MATERIALS AND METHODS.

After $24 \mathrm{hr}$ reaction, formic acid production became constant at 2.1 moles per mole of oligosaccharide and the amount of periodate consumed was 3.8 moles per mole of oligosaccharide.

In the oxidation of oligosaccharide $\mathrm{I}-3$, the amount of formic acid produced was 1.9 moles per mole of oligosaccharide and the amount of periodate consumed was 4.8 moles per mole

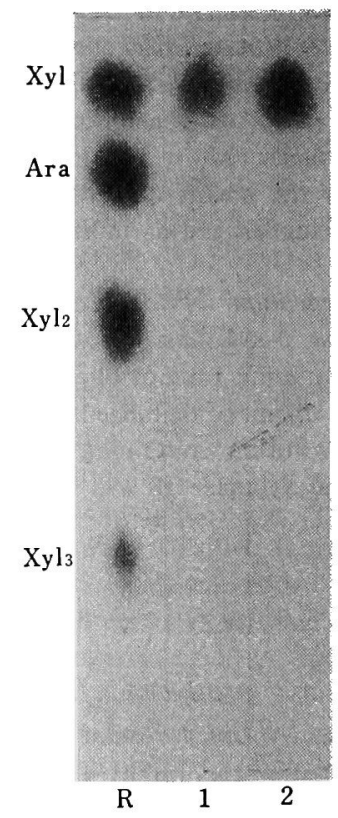

FIG. 3. Paper Chromatogram of Products from Reduced Oligosaccharide I-1 and I-3 by Hydrolysis with the $\beta$-Xylosidase.

After oligosaccharide I-1 and I-3 were reduced by sodium borohydride, the reduced oligosaccharide I-1 and I-3 were hydrolyzed by the $\beta$-xylosidase. See the text for the experimental details. Aniline hydrogen phthalate was used as detector in the paper chromatography.

$\mathrm{R}$ : reference sugars, 1: products from reduced oligosaccharide I-1, 2: products from reduced oligosaccharide I-3, Ara: arabinose, Xyl: xylose, $\mathrm{Xyl}_{2}$ : xylobiose, $\mathrm{Xyl}_{3}$ : xylotriose.

of oligosaccharide (Fig. 6).

Action of the xylanase II on phenyl $a-L_{-}$ arabinofuranoside and phenyl $\beta$ - $L$-arabinofuranoside

It was confirmed previously ${ }^{2}$ that the xylanase II can split both xylosidic and arabinosidic linkages of arabinoxylan. Thus, in order to clarify which linkage of $a$ - or $\beta$-Larabinofuranoside is split by this arabinofuranosidase activity of the xylanase II, the enzyme was allowed to react on phenyl $a$-Larabinofuranoside and phenyl $\beta$-L-arabinofuranoside. Reaction mixture containing $1 \mathrm{mg}$ of phenyl $a$-L-arabinofuranoside or phenyl $\beta$-L-arabinofuranoside which was dissolved in $0.1 \mathrm{ml}$ of $0.01 \mathrm{M}$ acetate buffer, $\mathrm{pH}$ 

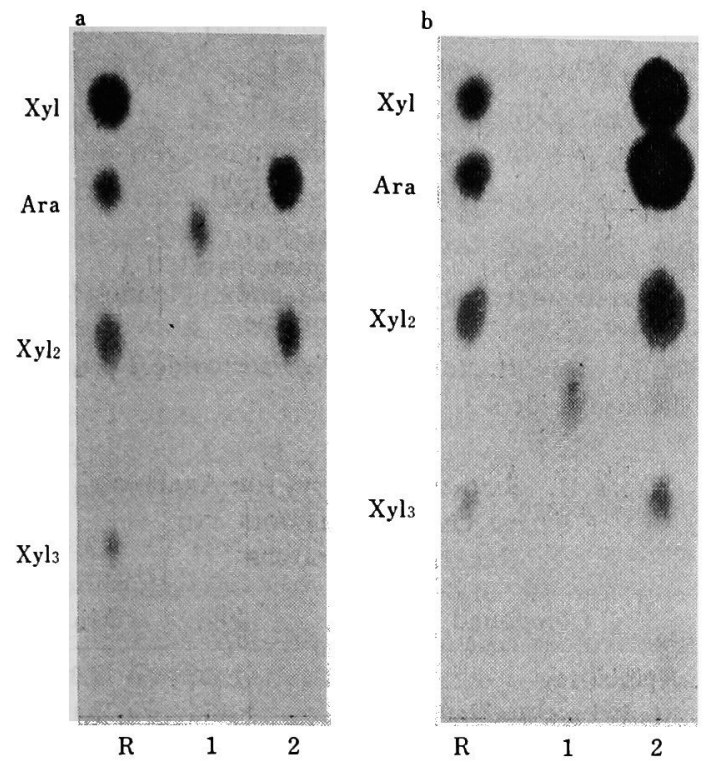

FIG. 4. Paper Chromatogram of Products from Oligosaccharide $\mathrm{I}-\mathbf{1}$ and $\mathrm{I}-\mathbf{3}$ by Hydrolysis with the Xylanase II.

a: Products of oligosaccharide I-1.

$\mathrm{R}$ : reference sugars, 1 : oligosaccharide $\mathrm{I}-1,2$ : products from oligosaccharide I-1 by hydrolysis with the xylanase II.

b: Products of oligosaccharide I-3.

$\mathrm{R}$ : reference sugars, 1 : oligosaccharide $\mathrm{I}-3,2$ : products from oligosaccharide I-3 by hydrolysis with the xylanase II.

Ara: arabinose, $\mathrm{Xyl}$ : xylose, $\mathrm{Xyl}_{2}$ : xylobiose, $\mathrm{Xyl}_{3}$ : xylotriose. See the text for experimental detail. Alkaline silver nitrate was used as a detector in the paper chromatography.

4.5 , and $0.1 \mathrm{ml}$ of enzyme solution containing $1 \mathrm{mg}$ of the xylanase II was incubated at $40^{\circ} \mathrm{C}$ for $24 \mathrm{hr}$. An arabinose formed was detected by paper chromatography. As shown in Fig. 5, the xylanase II splits preferentially $a$ L-arabinofuranosidic linkage.

\section{DISCUSSION}

From results shown in Fig. 1 and Table I, it appeared that the oligosaccharide $\mathrm{I}-1$ is trisaccharide consisting of one arabinose unit and two xylose units and the oligosaccharide I-3 is tetrasaccharide consisting of one arabinose unit and three xylose units. When these oligosaccharides were reduced at the reducing end by sodium borohydride

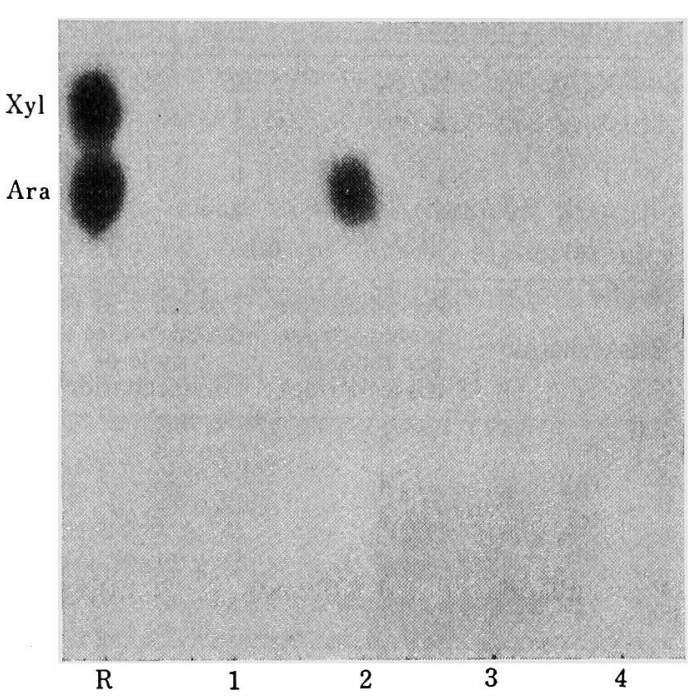

Fig. 5. Action of the Xylanase II on Phenyl- $a-\mathrm{L}-$ arabinofuranoside and Phenyl $\beta$-L-Arabinofuranoside.

Reaction mixture containing $1 \mathrm{mg}$ of phenyl $\alpha-\mathrm{L}$ arabinofuranoside or phenyl $\beta$-L-arabinofuranoside which was dissolved in $0.1 \mathrm{ml}$ of $0.01 \mathrm{M}$ acetate buffer, $\mathrm{pH} 4.5$, and $0.1 \mathrm{ml}$ of enzyme solution containing $1 \mathrm{mg}$ of the xylanase II was incubated at $40 \mathrm{C}$ for $24 \mathrm{hr}$. Aliquots of the mixture were spoted on Whatman No. 1 filter paper and chromatographed by the method described in the text. Aniline hydrogen phthalate was used as detector.

$\mathrm{R}$ : reference sugars, 1: phenyl $a$-L-arabinofuranoside, 2 : phenyl $a$-L-arabinofuranoside which incubated with the xylanase II, 3 : phenyl $\beta$-L-arabinofuranoside, 4: phenyl $\beta$-L-arabinofuranoside which incubated with the xylanase II. Ara: arabinose, $\mathrm{Xyl}$ : xylose.

and hydrolyzed with $1 \mathrm{~N}$ sulfric acid, in the hydrolyzates of reduced oligosaccharide I-1 and I-3, xylose and arabinose were detected in the proportion of $1: 1$ and $1: 2$, respectively. These facts show that both oligosaccharides lost one xylose residue by the reduction of the reducing ends and suggest that the reducing ends of the both oligosaccharides are xylose.

As shown in Fig. 4, the oligosaccharide I-1 gave arabinose and xylobiose in the proportion of $1: 1$, and the oligosaccharide $\mathrm{I}-3$ gave arabinose, xylose, xylobiose and xylotriose by the action of the xylanase II. In the case of oligosaccharide I-3, the fact seems to indicate the result that the oligosaccharide was at first hydrolyzed into arabinose and xylotriose by an arabinofuranosidic linkage splitting 
(a) Oligosaccharide I-1

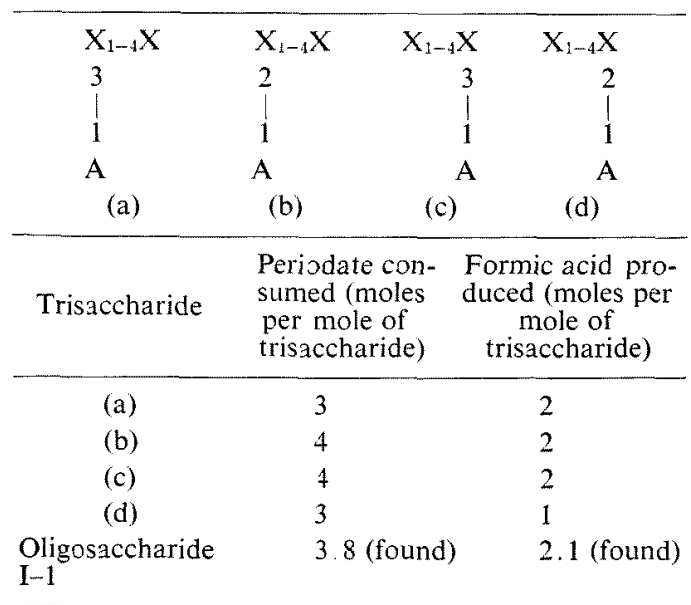

\begin{tabular}{|c|c|c|c|}
\hline \multicolumn{4}{|c|}{ (b) Oligosaccharide I-3 } \\
\hline$X_{1-4} X_{1-4} X$ & $X_{1-4} X_{1-4} X$ & $X_{1-4} X_{1-4} X$ & $\mathrm{X}_{1-4} \mathrm{X}_{1-4} \mathrm{X}$ \\
\hline 3 & 2 & 3 & 2 \\
\hline 1 & 1 & 1 & 1 \\
\hline A & A & A & A \\
\hline (a) & (b) & (c) & (d) \\
\hline $\mathrm{X}_{1-4} \mathrm{X}_{1-4} \mathrm{X}$ & $X_{1-4} X:-4$ & & \\
\hline 3 & 2 & & \\
\hline 1 & 1 & & \\
\hline A & A & & \\
\hline (e) & (f) & & \\
\hline
\end{tabular}

$\begin{array}{ccc}\text { Tetrasaccharide } & \begin{array}{c}\text { Periodate con- } \\ \text { sumed (moles per } \\ \text { mole of }\end{array} & \begin{array}{c}\text { Formic acid pro- } \\ \text { duced (moles per } \\ \text { mole of }\end{array} \\ \text { tetrasaccharide) } & \text { tetrasaccharide) }\end{array}$

$\begin{array}{cll}\text { (a) } & 4 & 2 \\ \text { (b) } & 5 & 2 \\ \text { (c) } & 5 & 3 \\ \text { (d) } & 5 & 3 \\ \text { (e) } & 5 & 2 \\ \text { (f) } & 4 & 1 \\ \text { Oligosaccharide } & 4.8 \text { (found) } & 1.9 \text { (found) } \\ \text { I-3 }\end{array}$

FIG. 6. Possible Structures of the Oligosaccharide I-1 and I-3.

activity of the xylanase II, and then the xylotriose was hydrolyzed into xylose and xylobiose by xylosidic linkage splitting activity of the xylanase II. Therefore, it seems that the oligosaccharide I-1 and the oligosaccharide I-3 have xylobiose and xylotriose, respectively, in their molecules as the backbone structures.

From these facts, as shown in Fig. 6

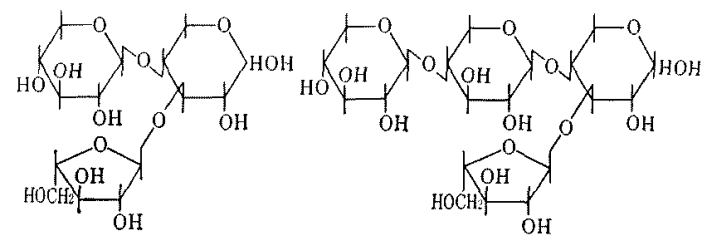

Oligosaccharide I-1

Oligosaccharide I-3 $3^{1} \alpha-L$-arabinofuranosyl- $\quad 3^{1}-\alpha-L-a r a b i n o f u r a n o s y l-$ xylobiose xylotriose

FIG. 7. The Structures of Oligosaccharide I-1 and Oligosaccharide I-3.

Table II. Rotatory Values for ArabinoseXYLOSE OLIGosacchaRIDES AND RELATED COMPOUNDS ${ }^{a}$;

\begin{tabular}{lcr}
\hline \multicolumn{1}{c}{ Compound } & {$[a]_{\mathrm{D}}$} & \multicolumn{1}{c}{$\mathrm{M}_{\mathrm{D}}$} \\
\hline 1. Xylobiose & $-26^{\circ}$ & $-7,330$ \\
2. Methyl $a$-L-arabinofuranoside & $-135^{\circ}$ & $-22,150$ \\
3. Methyl $\beta$-L-arabinofuranoside & $+118^{\circ}$ & $+19,350$ \\
$\quad$ Calc. for arabinose-xylose & & \\
$\quad$ trisaccharide & & \\
$\quad$ with $a$-L-arabinosyl unit $(1+2)$ & & $-29,480$ \\
$\quad$ with $\beta$-L-arabinosyl unit $(1+3)$ & & $+12,020$ \\
4. Oligosaccharide I-1 & $-80^{\circ}$ & $-33,120$ \\
5. Xylotriose & $-46.9^{\circ}$ & $-19,420$ \\
$\quad$ Calc. for arabinose-xylose & & \\
$\quad$ tetrasaccharide & & $-41,570$ \\
$\quad$ with $a$-L-arabinosyl unit $(5+2)$ & & -70 \\
$\quad$ with $\beta$-L-arabinosyl unit $(5+3)$ & & $-45,864$ \\
6. Oligosaccharide I-3 & $-84^{\circ}$ & $-4,50$
\end{tabular}

a) These data were cited from A. S. Perlin's data ${ }^{5}$ except compound $t$ and 6 .

several possible structures may be proposed for both oligosaccharides. The results of oxidation by sodium metaperiodate, however, suggest that the structure of oligosaccharide $\mathrm{I}-1$ is (b) or (c) in Fig. 6-a and that of oligosaccharide $\mathrm{I}-3$ is (b) or (e) in Fig. 6-b. In addition to the above results, the $\beta$-xylosidase produced only xylose as reduceing sugar from the both reduced oligosaccharides. It was confirmed previously that the $\beta$-xylosidase of Asp. niger split xylosidic linkage from the non reducing end of substrate. Thus it is considered that the resonable structure of the oligosaccharide $\mathrm{I}-1$ and $\mathrm{I}-3$ is correspond to (c) in Fig. 6-a and (e) in Fig. 6-b, respectively.

As shown in Table II, the calculated molecular rotation for the oligosaccharide I-1 is $-29,480$ in the case that an arabinosidic 
linkage is $\alpha$-configulation and in the case of $\beta$-configulation, the calculated value is $+12,020$. The calculated molecular rotation for the oligosaccharide I-3 is $-41,570$ in the case that an arabinosidic linkage is $a$-configulation and in the case of $\beta$-configulation, the calculated value is -70 .

The found molecular rotation of the oligosaccharide $\mathrm{I}-1$ is $-33,120$ (Table II, compound 4) and that of the oligosaccharide I-3 is -45,864 (Table II, compound 6).

Above facts suggest that the arabinosidic linkages of the both oligosaccharides are an $\alpha$-configulation. Moreover, since an arabinosidic linkage splitting activity of the xylanase II used in this experiment splits preferencially an $a$-L-arabinofuranosidic linkage (Fig. 5), it appears that the arabinosidic linkages of the oligosaccharide I-1 and oligosaccharide I-3 are an $a$-L-arabniofuranosidic linkage.

From these facts, it was suggested that the structure of the oligosaccharide I-1 is $3^{1}-a$ L-arabinofuranosylxylobiose and the structure of the oligosaccharide $\left[-3\right.$ is $3^{1}-a-L$-arabinofuranosylxylotriose (Fig. 7). Therefore, it appears that the oligosaccharide $\mathrm{I}-1$ is different from the trisaccharide isolated by C. T. Bishop or G. O. Aspinall and the oligosaccharide $\mathrm{I}-3$ is different from the tetrasaccharide isolated by H. R. Goldshmidt et al. Thus it appears that the mode of action of the xylanse I of $A s p$. niger is different from that of the enzyme preparations obtained from Myrothecium vercaria and Streptomyces.

The structures of two oligosaccharides consisting of arabinose and xylose units isolated from rice-straw arabinosylan suggest that the rice-straw arabinoxylan is composed of chain of 1,4-linked $\beta$-xylopyranose residues and some of them have side-chain to which is attached an L-arabniose residue by 1,3- $a$-furanosidic linkage.

Then, it leads to the assumption that the xylanase I of Asp. niger have a higher affinity to the xylosidic linkage of xylose residues which have an arabinofuranosyl branch than the xylosidic linkage of xylose residues that are devoid of an arabinofuranosyl branch in the rice-straw arabinoxylan.

Acknowledgement. The authors are grateful to Emeritus Prof. J. Fukumoto, Osaka City University, for his encouragement and support in this work. The authors are indebted to Prof. A. Kaji, Kagawa University for a gift of phenyl $a$-L-arabinofuranoside.

\section{REFERENCES}

1) J. Fukumoto, Y. Tsujisaka and S. Takenishi, Nippon Nogeikagaku Kaishi, 44, 447 (1970).

2) Y. Tsujisaka, S. Takenishi and J. Fukumoto, ibid., 45, 253 (1971).

3) C. T. Bishop, J. Am. Chem. Soc., 78, 2840 (1956).

4) G. O. Aspinall, I. M. Cairncross, R. J. Sturgeon and K. C. B. Wilkie, J. Chem. Soc., 1960, 3881.

5) H. R. Goldshmidt and A. S. Perlin, Can. J. Chem., 41, 2272 (1963).

6) S. Takenishi, Y. Tsujisaka and J. Fukumoto, J. Biochem., 73, 335 (1973).

7) A. E. Flood, E. L. Hirst and J. K. N. Jones, J. Chem. Soc, $1948,1679$.

8) T. E. Timell, Svensk Papperstid, 63, 668 (1960).

9) M. Dubois, K. A. Gilles, J. K. Hamilton, P. A. Rebers and F. Smith, Anal. Chem., 28, 350 (1956).

10) W. E. Trevelyan D. P. Procter and J. S. Harrison, Nature, 166, 444 (1950).

11) S. M. Partidge, ibid., 164, 443 (1949).

12) P. E. Fleury and J. Lange, J. Phrm. Chim., 17, 109 (1933); iden, ibid, 17, 196 (1933). 